\title{
Retrospective Comparative Study of Spontaneous Peritonitis Associated with Fungal and Bacterial Infection in Patients with Liver Cirrhosis
}

\section{Toru Shizuma*}

Department of Physiology, Tokai University School of Medicine, Japan

*Correspondence author: Toru Shizuma, Department of Physiology, Tokai University School of Medicine, 143, Shimokasuya, Isehara, Kanagawa 259-1193, Japan, Tel: +81-0463-93-1121; E-mail: shizuma@is.icc.u-tokai.ac.jp

Received date: February 10, 2018; Accepted date: February 19, 2018; Published date: February 27, 2018

Copyright: ( 2018 Shizuma T. This is an open-access article disturbed under the terms of the Creative Commons Attribution License, which permits unrestricted use, distribution, and reproduction in any medium, provided the original author and source are created.

\begin{abstract}
Objective: Although patients with liver cirrhosis (LC) have a high risk of developing bacterial and fungal infections, few studies have evaluated spontaneous fungal peritonitis (SFP) or fungiascites in this population. Accordingly, we conducted a retrospective comparative study of spontaneous peritonitis associated with fungal (SFP and fungiascites) or bacterial culture-positive ascites [spontaneous bacterial peritonitis (SBP) and bacterascites] in patients with LC.
\end{abstract}

Methods: This study enrolled 73 patients with LC and ascitic culture-positive spontaneous peritonitis, including four, three, 35, and 31 patients with SFP, fungiascites, culture-positive SBP, and bacterascites, respectively. We compared the laboratory findings, Child-Pugh scores, and 1-month mortality rates between patients with fungal disease, i.e., spontaneous peritonitis associated with fungal culture-positive ascites (SFP and fungiascites), and those with bacterial disease, i.e., spontaneous peritonitis associated with bacterial culture-positive ascites (culturepositive SBP and bacterascites).

Results: We observed no significant differences in the severity of underlying liver dysfunction and renal impairment between patients with fungal and bacterial disease. However, the 1-month mortality rate was significantly higher in patients with fungal disease than in those with bacterial disease $(71.4 \%, 5 / 7 \mathrm{vs} .25 .8 \%, 17 / 66 ; p=0.038)$.

Conclusion: In our retrospective study population, spontaneous peritonitis caused by fungi (SFP and fungiascites) was associated with a significantly higher short-term mortality rate compared with that of spontaneous peritonitis caused by bacteria (culture-positive SBP and bacterascites).

Further studies are required to investigate the underlying mechanisms and determine the effects of antifungal therapy on mortality.

Keywords Spontaneous fungal peritonitis; Fungiascites; Spontaneous bacterial peritonitis; Bacterascites, Ascites

Abbreviations: CNNA: Culture-Negative Neutrocytic Ascites; Cr: Creatinine; HRS: Hepatorenal Syndrome; LC: Liver Cirrhosis; MELD: Model For End-Stage Liver Disease; PMN: Polymorphonuclear Cell; PT: Prothrombin Time; SBP: Spontaneous Bacterial Peritonitis; SFP: Spontaneous Fungal Peritonitis

\section{Introduction}

Patients with liver cirrhosis (LC) are at a high risk of developing bacterial [1] and fungal infections [2] such as spontaneous bacterial peritonitis (SBP) or spontaneous fungal peritonitis (SFP), which can be life-threatening in this population [3]. The clinical features and prognosis of SBP, the most common type of bacterial infectious disease affecting patients with LC [4], have been well documented [3,5]. Furthermore, in bacterascites, a subtype of SBP, bacteria may be cleared naturally from ascitic fluid. Accordingly, the need for antibacterial therapies in such cases remains uncertain [6,7]. In contrast, the features of SFP (or fungiascites) in patients with LC remain less well-known, and few studies have compared SFP and SBP
[8-11] or, more generally, spontaneous peritonitis caused by fungi (SFP or fungiascites) with those caused by bacteria (SBP or bacterascites) [12]. To address this lack of evidence, we conducted a retrospective comparative study of spontaneous peritonitis caused by fungi vs. that caused by bacteria in a cohort of Japanese patients with LC according to the laboratory findings and short-term prognoses.

\section{Materials and Methods}

For this study, we enrolled 801 patients with LC and ascites who had undergone 1998 paracentesis procedures using standard techniques between 1996 and 2017. Of these procedures, 1608 were associated with both laboratory examinations and ascitic fluid cultures; these procedures could be further classified as 1496 hospitalizations and 112 outpatient procedures at three hospitals (Tokyo Women's Medical College, Nagashio Hospital, and International University of Health and Welfare Hospital) located in the Kanto district of Japan.

We excluded 10 cases involving primary or secondary prophylaxis for SBP prevention and 14 cases involving a diagnosis of SBP and follow-up paracentesis to predict early responses to initial treatment (usually performed 48 hours after the administration of antimicrobial agents against SBP) [3]. Following these exclusions, 781 patients with 
Page 2 of 4

LC and 1584 paracentesis procedures were included in the study (1474 were hospitalized, 110 were treated as outpatients). Of these 781 subjects, $483(61.8 \%)$ were male and $298(38.2 \%)$ were female, with a mean age of 64.3 (range, 36-87) years at the time of paracentesis. The hepatitis $B$ surface antigen and hepatitis $C$ virus antibody positivity rates were $10.4 \%(81 / 781)$ and $63.5 \%(496 / 781)$, respectively.

\section{Diagnosis of spontaneous peritonitis}

Cases of infectious pleural effusion, peritonitis carcinomatosa, and hemorrhagic ascites were excluded from the diagnosis of spontaneous peritonitis (SBP, bacterascites, SFP, and fungiascites). We additionally excluded secondary bacterial peritonitis, which comprised ascitic fluid infection attributed to an intraabdominal infection (e.g., perforation of the gastrointestinal tract).

Culture-positive SBP and bacterascites were classified as spontaneous peritonitis with a bacteria-positive ascitic culture. SBP was diagnosed if the polymorphonuclear cell (PMN) count in the ascitic fluid was $\geq 250 / \mathrm{mm}^{3}$, regardless of the presence of bacteria in the ascitic fluid [13]. However, culture-negative neutrocytic ascites (CNNA) was excluded from the category of spontaneous peritonitis with bacteria-positive ascitic culture in this study. Bacterascites was diagnosed when the PMN cell count was $<250 / \mathrm{mm}^{3}$ and bacteria were isolated from the ascitic fluid [13].

SFP and fungiascites were classified as spontaneous peritonitis with a fungus-positive ascitic culture. SFP was diagnosed if the PMN count in the ascitic fluid was $\geq 250$ cells $/ \mathrm{mm}^{3}$ and a fungal culture was positive, regardless of bacterial co-colonization $[3,9]$. Fungiascites (i.e., fungal ascites) was defined as a fungus-positive ascites culture (regardless of bacterial co-colonization) and a PMN count of $<250$ cells $/ \mathrm{mm}^{3}[3,9]$.

\section{Laboratory analyses of sera and ascites fluid}

We retrospectively examined various serum laboratory findings [ prothrombin time (PT\%), albumin levels, total bilirubin levels, creatinine (Cr) levels ], the Child-Pugh scores, and total protein levels in the ascitic fluid at the time of paracentesis and compared these findings between patients with fungal disease, i.e., spontaneous peritonitis associated with fungal culture-positive ascites (SFP and fungiascites), and those with bacterial disease, i.e., spontaneous peritonitis associated with bacterial culture-positive ascites (culturepositive SBP and bacterascites).

\section{Comparison of Short-Term Prognosis}

We retrospectively examined the short-term mortality (up to 1 month after paracentesis) between patients with fungal disease and those with bacterial disease.

\section{Statistical Analysis}

The data were analyzed using the Statistical Package for Social Sciences, version 20 (SPSS, Inc., Chicago, IL, USA). A chi-square test was used to compare the 1-month mortality rates between patients with fungal disease and those with bacterial disease. The unpaired ttest was used to compare the serological and ascitic laboratory findings and Child-Pugh scores between the groups. All values were presented as mean \pm standard deviations, and $\mathrm{p}<0.05$ was considered statistically significant.

\section{Results}

\section{Incidence of spontaneous peritonitis}

Among 86 cases of SBP in our sample, 40.7\% (35/86) had culturepositive SBP and $59.3 \%(51 / 86)$ had CNNA. The frequencies of culture-positive SBP and bacterascites were $47.9 \%$ (35/73) and $42.5 \%$ (31/73), respectively, among all cases of ascitic culture-positive spontaneous peritonitis. Regarding fungal disease, the frequencies of SFP and fungiascites were 5.5\% (4/73) and 4.1\% (3/73), respectively, among all cases of ascitic culture-positive spontaneous peritonitis.

\section{Comparison of serological and ascitic laboratory findings}

Table 1 shows the comparison of the serological laboratory findings, Child-Pugh scores, and total protein levels in the ascitic fluid between subjects with fungal spontaneous peritonitis and those with ascitic culture-positive bacterial spontaneous peritonitis. There were no significant differences in the PT, serum albumin levels, total bilirubin levels, Cr levels, Child-Pugh scores, and total protein levels in the ascitic fluid between the two groups.

\section{Prognosis of spontaneous peritonitis}

One-month mortality rates of $31.4 \%$ (11/35), 19.4\% (6/31), 100\% $(4 / 4)$, and $33.3 \%(1 / 3)$ were observed for culture-positive SBP, bacterascites, SFP, and fungiascites, respectively. Furthermore, three of four SFP patients died before receiving a diagnosis of SFP, and all died within 2 weeks after paracenteses. The 1 -month mortality rate of ascites culture-positive fungal spontaneous peritonitis was significantly higher than that of ascites culture-positive bacterial spontaneous peritonitis $(71.4 \%, 5 / 7$ vs. $25.8 \%, 17 / 66 ; \mathrm{p}=0.038)$ (Table 1$)$.

\begin{tabular}{|l|l|l|l|}
\hline Variables & $\begin{array}{l}\text { Fungal spontaneous } \\
\text { peritonitis (n=7) }\end{array}$ & $\begin{array}{l}\text { Bacterial } \\
\text { spontaneous } \\
\text { peritonitis (n=66) }\end{array}$ & p-value \\
\hline Child-Pugh class & $0 / 7(0.0 \%)$ & $0 / 66(0.0 \%)$ & \\
\hline A & $0 / 7(0.0 \%)$ & $4 / 66(6.1 \%)$ & 0.185 \\
\hline B & $7 / 7(100.0 \%)$ & $62 / 66(93.9 \%)$ & 0.245 \\
\hline C & $12.7 \pm 1.30$ & $12.1 \pm 1.28$ & 0.689 \\
\hline $\begin{array}{l}\text { Child-Pugh score } \\
\text { Serum albumin }\end{array}$ & $2.30 \pm 0.32$ & $2.45 \pm 0.32$ & 0.137 \\
\hline $\begin{array}{l}\text { Serum } \\
\text { bilirubin (mg/dL) }\end{array}$ & $3.7 \pm 1.3$ & $1.25 \pm 0.25$ & $0.4 \pm 1.9$ \\
\hline $\begin{array}{l}\text { Serum creatinine } \\
\text { (mg/dL) }\end{array}$ & $1.27 \pm 0.36$ & $46.0 \pm 5.9$ & 0.36 \\
\hline $\begin{array}{l}\text { Prothrombin time } \\
\text { (\%) }\end{array}$ & $42.4 \pm 6.1$ & $25.8 \%(17 / 66)$ & \\
\hline $\begin{array}{l}\text { Total protein in } \\
\text { the ascitic fluid } \\
\text { (g/dL) }\end{array}$ & $1.31 \pm 0.24$ & & 0.138 \\
\hline $\begin{array}{l}\text { One-month } \\
\text { mortality rate }\end{array}$ & $71.4 \%(5 / 7)$ & & \\
\hline
\end{tabular}

Table 1: Comparison between fungal spontaneous peritonitis and bacterial spontaneous peritonitis in patients with liver cirrhosis. 


\section{Discussion}

Fungal infections are usually observed in immune compromised conditions such as in transplant patients and those with malignancies, and it is well-known that patients with LC are also susceptible to microbial infection including fungal disease [2]. A recent large-scale multi-center study of patients with LC and concomitant infection found that $12.7 \%(134 / 1052)$ of such cases involved fungal infection [2], indicating that fungal infections are not uncommon in these patients. Factors such as nosocomial infection, a low protein concentration in the ascitic fluid, higher Model for End-Stage Liver Disease (MELD) score, and occurrence of hepatorenal syndrome (HRS) have been associated with the incidence of fungal infection in patients with LC $[2,14]$. Moreover, the use of prophylactic antibiotics or antibiotics for bacterial infections such as SBP has been estimated to be a risk factor for fungal infections such as SFP [2]. A recent study by Bajaj et al. [15] indicated that the use of antibiotics induced a significant change of fungal dysbiosis in patients with LC, and excessive growth of Candida species was observed after antibiotics use in these patients, indicating that antibiotic use may induce creation of ideal conditions for these subsequent fungal infections. Incidence of fungal infections may also be associated with an immunosuppressed state including intestinal environment in patients with advanced LC that is exacerbated by prior bacterial infections $[2,16]$. Impairment of intestinal barrier function such as high intestinal permeability, which is more common in advanced LC, may also facilitate fungal translocation across the gut mucosa and incidence of fungal infections $[9,17]$. Immunosuppression and malnutrition in LC patients also promote this fungal translocation [18]. Similarly, Bajaj et al. [2] identified age, complications of acute kidney injury, and a high MELD score as independent risk factors for mortality in patients with LC and fungal infections and observed a high rate of mortality in this population despite treatment with antifungal agents after diagnosis [2]. Consistent with our findings, Alexopoulou et al. [11] reported significantly higher 1-and 6-month mortalities among patients with LC and concomitant fungal infections compared with those among patients with LC and bacterial infections.

Spontaneous peritonitis caused by fungi includes SFP and fungiascites. Bajaj et al. [2] further reported that $6.0 \%$ (8/134) of fungal infections in patients with LC involved spontaneous peritonitis [2]. The increased risk of fungal peritonitis among patients with LC may be partly attributed to the use of antibiotics against SBP. These abovementioned drugs can adversely allow excessive fungal growth in the intestinal flora, and the subsequent translocation of fungi into the peritoneal cavity can induce SFP or fungiascites [18].

The early diagnosis of such cases is challenging, partially because the extended time required for fungal growth in a conventional microbial culture slows the diagnostic process [19]. Moreover, the usefulness of modalities, such as polymerase chain reaction or assays for the fungal biomarker 1,3-beta-d-glucan in the ascitic fluid for the diagnosis of SFP or fungiascites, has not been well established $[10,19]$, although a previous report of a case of SFP described elevated levels of 1,3-beta-d-glucan in the ascitic fluid [20].

To date, few studies have focused on fungiascites. For example, in a series of nine cases of fungiascites, Bucsics et al. [12] reported that seven cases and two cases exhibited a Child-Pugh liver dysfunction class of B and C, respectively; furthermore, four cases (44\%) harbored bacterial co-infections, and the in-hospital mortality was 33\% (3/9). Park et al. [21] evaluated 16 cases of spontaneous peritonitis wherein Cryptococcus was identified in the ascitic fluid, among which 13 cases
(81\%) were reported to be involved fungiascites (PMN count in the ascitic fluid $\left.<250 / \mathrm{mm}^{3}\right)$. They also reported that $69 \%(11 / 16)$ of patients with spontaneous peritonitis cases involving Cryptococcus died within 1 week after paracentesis [21].

Despite a recent increase in research interest, few studies have targeted SFP. Of these, several have identified high Child-Pugh and MELD scores $[9,17,18]$, invasive procedures [8], a longer hospital stay $[8,22]$, and nosocomial infection $[9,22]$ as risk factors for the incidence of SFP. Similarly, studies have identified severe underlying liver disease [9], a high Child-Pugh [10] or MELD score [19], use of antibacterial prophylaxis [18], the incidence of HRS [18], a low protein concentration in the ascitic fluid [18], high Acute Physiology And Chronic Health Evaluation (APACHE) II score [19], and incidence of septic shock as risk factors associated with SFP-related hospital mortality in SFP [8]. Furthermore, several studies have identified Candida albicans as the most frequent isolate among SFP cases $[8-10,12,17-19,22]$ and reported polymicrobial infection (e.g., bacterial co-colonization) in $32-74 \%$ of cases $[8,9,12,19,22]$.

In our study, we observed no significant differences in various laboratory values or Child-Pugh scores between patients with spontaneous peritonitis associated with fungal culture-positive ascites (SFP and fungiascites) and those with spontaneous peritonitis associated with bacterial culture-positive ascites (culture-positive SBP and bacterascites). As noted, few previous studies have directly compared the serum and ascitic findings between these groups of patients [12] or the conditions of SFP and SBP [8-11]. Consistent with our findings, previous comparative studies reported no significant differences in PT (or the International Normalized Ratio; INR) or the albumin and bilirubin levels between cases of spontaneous peritonitis caused by fungi and those caused by bacteria [12], between cases of SFP and SBP [8], or between cases of SFP and CNNA [10]. The data concerning serum $\mathrm{Cr}$ levels have been less consistent; although some studies reported no significant differences in serum $\mathrm{Cr}$ levels between spontaneous peritonitis caused by fungi and those caused by bacteria [12] or between SFP and SBP [8,9], Elkhateeb et al. [10] reported that the serum Cr levels were significantly higher in SFP than in CNNA. Regarding underlying disease severity, Gravito-Soares et al. [8] reported no significant differences in Child-Pugh and MELD scores between SFP and SBP, and Bucsics et al. [12] reported no significant differences in Child-Pugh scores between spontaneous peritonitis caused by fungi and that caused by bacteria, which was consistent with our findings. In contrast, Hwang et al. [9] reported that the ChildPugh scores were significantly higher in SFP than in SBP. Regarding ascitic findings, previous comparative studies observed no significant differences in total protein or albumin between SFP and SBP $[8,11]$ or between SFP and CNNA [10], which was consistent with our study.

Previous studies reported worse rates of SFP mortality relative to our findings, with estimates of $56-90 \%[3,8,9,17,19]$. SFP is only diagnosed after fungal species have been identified in an ascitic fluid culture. This delay in diagnosis contributes to the increased mortality associated with this condition, although a lack of suspicion of SFP and delay in administration of antifungal therapy are also factors $[9,10,17,18,22]$. In previous studies, Gravito-Soares et al. [8] reported a significantly higher 30 -day mortality among SFP cases $(50 \%, 4 / 8)$ relative to SBP cases $(24.4 \%, 29 / 119)$, and Hwang et al. [9] reported significantly higher 1- and 6-month mortalities of SFP (73.3\%, 11/15 and $93.3 \%, 14 / 15$, respectively) compared with those of SBP $(28.7 \%$, $115 / 401$ and $56.1 \%, 225 / 401$, respectively), which was consistent with our findings. In contrast, Bucsics et al. [12] reported no significant 
differences in the in-hospital mortality between patients with spontaneous peritonitis caused by fungi (SFP or fungiascites) $(35.7 \%$, $5 / 14)$ and those with spontaneous peritonitis caused by bacteria (culture-positive SBP or bacterascites) $(25.9 \%, 29 / 112)$. However, in our study, the short-term mortality was higher for spontaneous peritonitis caused by fungi (SFP and fungiascites) than that for spontaneous peritonitis caused by bacteria (culture-positive SBP and bacterascites). These discrepancies in mortality outcomes may be partly attributable to the ratio of SFP among all cases of spontaneous peritonitis caused by fungi. The frequency of SFP was $35.7 \%(5 / 14)$ in a previous study by Bucsics et al. [12] and 71.4\% (5/7) in our study. Furthermore, all four patients with SFP in our study died within 2 weeks after paracenteses.

It remains uncertain whether antifungal therapy would decrease the mortality rate associated with spontaneous peritonitis caused by fungi [23]. Previous studies suggest that directed antifungal therapy may not improve the outcomes of some patients with SFP [19,22], and this is partly attributed to the previous administration of empirical treatment for SBP and delays in initiating antifungal therapy [22]. Therefore, further research is needed to determine the efficacy of treatments for SFP or fungiascites.

\section{Conclusion}

In our sample of patients with LC, spontaneous peritonitis caused by fungi (SFP and fungiascites) was associated with a significantly higher 1-month mortality rate compared with that of spontaneous peritonitis caused by bacteria (culture-positive SBP and bacterascites). However, further research is needed to clarify the features of SFP and fungiascites and determine the best treatment options.

\section{References}

1. Bunchorntavakul C, Chavalitdhamrong D (2012) Bacterial infections other than spontaneous bacterial peritonitis in cirrhosis. World J Hepatol 4: 158-168.

2. Bajaj JS, Rajender Reddy K, Tandon P, Wong F, Kamath PS, et al. (2017) Prediction of fungal infection development and their impact on survival using the NACSELD Cohort. Am J Gastroenterol: [Epub ahead of print].

3. Fiore M, Maraolo AE, Leone S, Gentile I, Cuomo A, et al. (2017) Spontaneous peritonitis in critically ill cirrhotic patients: a diagnostic algorithm for clinicians and future perspectives. Ther Clin Risk Manag 13: 1409-1414.

4. Fernández J, Navasa M, Gómez J, Colmenero J, Vila J, et al. (2002) Bacterial infections in cirrhosis: epidemiological changes with invasive procedures and norfloxacin prophylaxis. Hepatology 35: 140-148.

5. Wiest R, Krag A, Gerbes A (2012) Spontaneous bacterial peritonitis: recent guidelines and beyond. Gut 61: 297-310.

6. Shizuma $T$ (2014) Retrospective Investigation of Bacterascites and Spontaneous Bacterial Peritonitis in Liver Cirrhosis Patients Undergoing Paracentesis. J Clin Trials 4: 166
7. Pelletier G, Lesur G, Ink O, Hagege H, Attali P, et al. (1991) Asymptomatic bacterascites: is it spontaneous bacterial peritonitis? Hepatology 14: 112-115.

8. Gravito-Soares M, Gravito-Soares E, Lopes S, Ribeiro G, Figueiredo P (2017) Spontaneous fungal peritonitis: a rare but severe complication of liver cirrhosis. Eur J Gastroenterol Hepatol 29: 1010-1016.

9. Hwang SY, Yu SJ, Lee JH, Kim JS, Yoon JW, et al. (2014) Spontaneous fungal peritonitis: a severe complication in patients with advanced liver cirrhosis. Eur J Clin Microbiol Infect Dis 33: 259-264.

10. Elkhateeb SR, Gouda NS, Ibrahim AS, Anwar R, Eissa LA (2017) PCRdetected fungal infection is associated with fatal outcomes in cirrhotic patients with spontaneous peritonitis. Egyptian Journal of Basic and Applied Sciences: 4: 42-46.

11. Alexopoulou A, Vasilieva L, Agiasotelli D, Dourakis SP (2015) Fungal infections in patients with cirrhosis. J Hepatol 63: 1043-1045.

12. Bucsics T, Schwabl P, Mandorfer M, Peck-Radosavljevic M (2016) Prognosis of cirrhotic patients with fungiascites and spontaneous fungal peritonitis (SFP). J Hepatol 64: 1452-1454.

13. Rimola A, García-Tsao G, Navasa M, Piddock LJ, Planas R, et al. (2000) Diagnosis, treatment and prophylaxis of spontaneous bacterial peritonitis: a consensus document. International Ascites Club. J Hepatol 32: 142-153.

14. Hassan EA, Abd El-Rehim AS, Hassany SM, Ahmed AO, Elsherbiny NM, Mohammed MH (2014) Fungal infection in patients with end-stage liver disease: low frequency or low index of suspicion. Int J Infect Dis 23: 69-74.

15. Bajaj JS, Liu EJ, Kheradman R, Fagan A, Heuman DM, et al. (2017) Fungal dysbiosis in cirrhosis. Gut: [Epub ahead of print].

16. Shawcross DL (2015) Is it time to target gut dysbiosis and immune dysfunction in the therapy of hepatic encephalopathy? Expert Rev Gastroenterol Hepatol 9: 539-542.

17. Lahmer T, Brandl A, Rasch S, Schmid RM, Huber W (2016) Fungal Peritonitis: Underestimated Disease in Critically Ill Patients with Liver Cirrhosis and Spontaneous Peritonitis. PLoS One 11: e0158389.

18. Fiore M, Leone S (2016) Spontaneous fungal peritonitis: Epidemiology, current evidence and future prospective. World J Gastroenterol 22: 7742-7747.

19. Bremmer DN, Garavaglia JM, Shields RK (2015) Spontaneous fungal peritonitis: a devastating complication of cirrhosis. Mycoses 58: 387-393.

20. Majeed A, Ullah W, Zahid U, Al Mohajer M (2016) Persistent spontaneous fungal peritonitis secondary to Candida albicans in a patient with alcoholic cirrhosis and review of the literature. BMJ Case Rep 2016.

21. Park WB, Choe YJ, Lee KD, Lee CS, Kim HB, et al. (2006) Spontaneous cryptococcal peritonitis in patients with liver cirrhosis. Am J Med 119: 169-171.

22. Fiore M, Chiodini P, Pota V, Sansone P, Passavanti MB, et al. (2017) Risk of spontaneous fungal peritonitis (SFP) in hospitalized cirrhotic patients with ascites: a systematic review of observational studies and metaanalysis. Minerva Anestesiol 83: 1309-1316.

23. Dubler S, Laun M, Koch C, Hecker A, Weiterer S, et al. (2017) The impact of real life treatment strategies for Candida peritonitis-A retrospective analysis. Mycoses 60: 440-446. 